\title{
The Effect of Feedback and Information on Children's Pedometer Step Counts at School
}

\section{Zoe Butcher, Stuart Fairclough, Gareth Stratton, and David Richardson}

\begin{abstract}
This study examined whether feedback or feedback plus physical activity information could increase the number of pedometer steps taken during 1 school week. One hundred seventy-seven students (mean age $9.124 \pm 1.11$ years) in three elementary schools participated. Schools were randomly assigned to control $(\mathrm{CON})$, feedback $(\mathrm{FB})$, or feedback plus information $(\mathrm{FB}+\mathrm{I})$ groups. Children wore pedometers during school time for 5 consecutive weekdays. The total steps of the groups were recorded at the end of each school day, with students in the FB and $\mathrm{FB}+\mathrm{I}$ groups free to view their step counts. In addition, the FB+I group received information and ideas about how they could increase their daily steps. The CON group received no step-count feedback or information. Students in the FB+I group achieved significantly more steps per minute $(17.17 \pm 4.87)$ than those in the FB $(13.77 \pm 4.06, p=0.003)$ and $\mathrm{CON}(12.41 \pm 3.12, p=0.0001)$ groups. Information, as well as step-count feedback, increased elementary students' school-based physical activity (number of steps) in the short term. A longer intervention period is necessary to assess the sustained impact of this type of approach.
\end{abstract}

\section{Key Words:}

The health benefits of physical activity (PA) during childhood have been clearly established $(6,3)$. These benefits can influence adult health status, with evidence now accumulating to suggest that the onset of many chronic diseases such as obesity, Type II diabetes, cardiovascular disease (CVD), and some cancers begins in childhood $(16,27,12)$. In response to this and other evidence, physical activity guidelines recommend that elementary schoolchildren should accumulate 60 min or more of moderate- to vigorous-intensity physical activity (MVPA) per day (6). It is widely thought, however, that children do not meet these guidelines (8) and that this situation gets worse as children progress through adolescence $(13,22)$.

Butcher and Fairclough are with the School of Physical and Outdoor Education, Stratton and Richardson are with the Research Institute for Sport \& Exercise Sciences, Liverpool John Moores University, Liverpool, UK L2 3ET.. 
Children are a captive audience in schools because they typically spend $6 \mathrm{hr}$ per day, 5 days a week, for approximately 40 weeks of the year in this environment. Moreover, schools have an infrastructure of trained educators, facilities, and spaces to be active and so are logical environments for promoting PA among children $(11,18,19)$. From a health-promotion perspective, this is important because lifestyle and risk behaviors related to PA and chronic diseases can be learned early at school (25).

Although schools provide a promising environment for PA engagement, this does not guarantee that children will maximize these opportunities throughout the school day. Thus, it is important to identify strategies to motivate children to develop and maintain appropriate PA behaviors. In order to maintain and increase children's PA, interventions should enhance competence perceptions, social support, and enjoyment (31). Support for this view is provided through Harter's model of global self-worth, which suggests that perceived competence, self-worth, and enjoyment mediate PA behavior (14). Improving competence is vital because experiences of success in PA contexts will lead to increased intrinsic motivation and enjoyment (10). For children to develop perceived competence they need information on their physical achievement (31). One way to provide this is to give them task-specific feedback that can be used to facilitate improved performance (7).

One identified method of providing feedback on PA is the use of pedometers (28). Pedometers offer a simple, relatively inexpensive, reliable, and valid measure of PA (29). They can be used to provide an immediate and continuous measure of PA, as well as an environmental cue to be active (28). From an educational perspective, schools might find them to be attractive tools for measuring and promoting student PA. Beighle and colleagues (2) advocate using pedometers to provide feedback on the number of daily steps taken. When used in combination with goal-setting strategies, such feedback could be used as a motivational tool to increase PA. To our knowledge, only one previous study has investigated the motivational effect of pedometers on the number of steps taken. Shomaker (27) conducted the study during a walking-for-fitness class on a sample of 45 adults. The control group wore sealed pedometers, whereas members of the treatment group were able to view their accumulated steps. It was concluded that the step-count feedback appeared to facilitate the treatment group in maintaining a constant rate of steps/min over time compared with the control group.

The present study examined the effects of providing elementary schoolchildren with feedback and information relating to the number of pedometer steps accumulated during a full school day. The primary hypothesis was that children who received step-count feedback would increase their daily steps on subsequent days compared with children who received no feedback. The secondary hypothesis was that children who received step-count feedback combined with information and ideas about how to be more active would increase their daily steps on subsequent days more than children who received feedback only or no feedback at all. Third, the study aimed to examine the day-to-day differences between the treatment groups. The final aim was to investigate any gender differences in step counts. 


\section{Methods}

\section{Participants and Settings}

Students were recruited from three schools in Liverpool, England. Ten schools were randomly selected and approached with the research proposal, and three agreed to participate. The three schools were comparable in size and situated in similar areas of social and economical disadvantage, with over $50 \%$ of children per school entitled to free school meals (20). All students in Grades 3-6 (ages 7-11 years) from each school were invited to take part. Informed consent was obtained from the parents/guardians of 177 children, giving a participation rate of $45 \%$. Ethical approval was obtained from the Liverpool John Moores University Ethics Committee.

Each school was randomly assigned to one of the three intervention conditions: control (CON; males $n=18$, females $n=21$ ), feedback (FB; males $n=17$, females $n=35$ ), and feedback plus information ( $\mathrm{FB}+\mathrm{I}$; males $n=25$, females $n$ =25). Data were collected in each school for 1 school week between December 2004 and April 2005. School days began at 9 a.m. each day and ended at 3 p.m. in the FB+I school and 3:30 p.m. in the other two schools.

All schools had set recess periods, with the first occurring in the morning for $15 \mathrm{~min}$ and the second at lunchtime, lasting for $1 \mathrm{hr}$. The FB+I school also had an additional 15-min recess period in the afternoon. All students were timetabled for $1 \mathrm{hr}$ of physical education (PE) per week.

\section{Instruments and Procedures}

Stature was measured to the nearest $1 \mathrm{~mm}$ using a Leicester height measure (Birmingham, England), and body mass was calculated to the nearest $100 \mathrm{~g}$ using Seca weighing scales (Birmingham, England) (17). Body-mass index was calculated as $\mathrm{kg} / \mathrm{m}^{2}$.

Each student wore a calibrated (30) Ez-V digital pedometer (RYP Sports, USA) on the waistband above the right hip in order to measure the number of steps taken during the school day. The first author validated this budget pedometer with 18 students (mean age $=8.6 \pm 0.5$ years, stature $=1.4 \pm 0.1 \mathrm{~m}$, and body mass $=$ $30.8 \pm 5.7 \mathrm{~kg}$ ) according to the treadmill protocol outlined by Beets et al. (1). High interobserver agreement (mean $\mathrm{ICC}=.94,95 \% \mathrm{CI}=0.772-0.988$ ) was established, and moderate to high agreement was found between pedometer and observed steps (mean ICC $=.897,95 \% \mathrm{CI}=0.724-0.961$ ). The CON-group students wore sealed pedometers so that they were unable to see how many steps they had taken. At the end of each day a member of the research team retrieved the pedometers, and the total steps were recorded without being revealed to the students. Pedometers were then set to zero and resealed before the next day's recording. Students in the FB group wore unsealed pedometers and so were free to look at the display and obtain feedback on the number of steps they had taken. At the end of each day students in the FB group recorded the number of steps on personalized record sheets. To ensure that steps were recorded accurately, the research team and the class teachers monitored this process. The FB group was asked to look at their step totals 
and attempt to increase them during the following day. Pedometers were then reset and removed in preparation for the next day, when each child would wear the same one. The FB+I group followed the same procedure as the FB students, except they also received information and ideas on how they could increase their daily steps. Both FB+I students and their teachers were made aware of the different opportunities in the school day for physical activity, and then suggestions were made on how to maximize these opportunities. Students were encouraged to take part in activities as much as possible during classroom-based lessons, PE classes, and on the playground. They were also told how helping the teachers with setting up equipment, running errands, and getting changed quickly for PE would all help increase their accumulated step counts. For this reason, the teachers were asked to positively reinforce the benefits of PA to the students. Teachers were also provided with some simple ways they could facilitate increased PA among their classes. These involved incorporating activity breaks in the classroom, facilitating active recess by providing equipment and structured activities, not withholding recess or $\mathrm{PE}$ as punishment, and making PE lessons more efficient by increasing the time that students have to actively practice, play, and perform.

Pedometer steps were recorded at the end of each day. Counts below 1,000 or above 30,000 steps were deleted and treated as missing data (26). Missing data were then replaced with the child's own mean weekly end-of-day score, following procedures outlined by Kang et al. (15). In order to account for the differing lengths of the school day in the respective schools, end-of-day pedometer counts were divided by the number of minutes in the school day to create the dependent variable of pedometer steps/min.

\section{Statistical Analyses}

The data were first assessed for normality, and any outliers were checked for faulty measurements. Initial analyses were conducted using one-way analysis of variance (ANOVA) to explore any differences between the anthropometric characteristics of the three groups. For the main analysis a repeated-measures ANCOVA with body-mass index as a covariate was performed to explore the differences in daily pedometer steps/min by day and between condition and gender groups. GamesHowell post hoc analyses were conducted to identify between-conditions group differences. All analyses were performed using SPSS for Windows ${ }^{\circledR}$ Version 12.0 (SPSS Inc., Chicago, IL), and statistical significance was set to $p<.05$.

\section{Results}

Data were obtained from 177 students from the three different schools. Thirty-six students did not record sufficient pedometer data, with 4 of these having their pedometers confiscated by staff for misuse. After data cleaning, 141 students recorded usable pedometer data. Of these students 39 were from the CON group, 52 were in the FB group, and 50 were from the FB+I group. There were no significant group differences in stature, mass, or body-mass index (Table 1). The mean body-mass index of all three groups fell into the age- and sex-specific category for normal body mass, but of the whole sample, 39 children were deemed to be overweight or obese (5). 
The ANCOVA revealed a significant main effect for treatment group, $F(2$, $141)=11.7, p=.0001$, and gender, $F(1,141)=13.0, p=0.0001$. Post hoc analyses indicated that there was a significant difference in daily pedometer steps $/ \mathrm{min}$ between the $\mathrm{CON}$ and $\mathrm{FB}+\mathrm{I}(p=.0001)$ groups and between $\mathrm{FB}$ and $\mathrm{FB}+\mathrm{I}(p=$ .003 ), but not between CON and FB groups (Figure 1). Furthermore, boys accumulated significantly more steps/min than girls, $F(139,141)=3.2, p=.001$. A significant interaction was noted between day and treatment group, $F(6.8,144)=$ $6.0, p=.0001$ ), but not between gender and treatment group.

Figure 2 illustrates the pedometer steps/min for each treatment group for each day of the data-collection period. Pairwise comparisons revealed significant dif-

\section{Table 1 Anthropometric Characteristics of Students in Order of Treatment Group}

\begin{tabular}{lccccccccc}
\hline & \multicolumn{2}{c}{ Control } & \multicolumn{1}{c}{ FB } & \multicolumn{3}{c}{ FB+l } & & \\
\cline { 2 - 7 } & $\boldsymbol{M}$ & $\boldsymbol{S D}$ & $\boldsymbol{M}$ & $\boldsymbol{S D}$ & $\boldsymbol{M}$ & $\boldsymbol{S} \boldsymbol{D}$ & $\boldsymbol{F}$ & $\boldsymbol{p}$ \\
\hline Stature (m) & 1.37 & 0.08 & 1.35 & 0.09 & 1.38 & 0.09 & 1.56 & .21 \\
Mass (kg) & 35.18 & 9.31 & 3.4 & 8.7 & 35.34 & 10.8 & 0.28 & .76 \\
$\begin{array}{l}\text { Body-mass index } \\
\left(\mathrm{kg} / \mathrm{m}^{2}\right)\end{array}$ & 18.45 & 3.21 & 18.45 & 3.4 & 18.26 & 3.74 & 0.05 & .95 \\
\hline
\end{tabular}

Note. $\mathrm{FB}=$ feedback; $\mathrm{FB}+\mathrm{I}=$ feedback plus information.

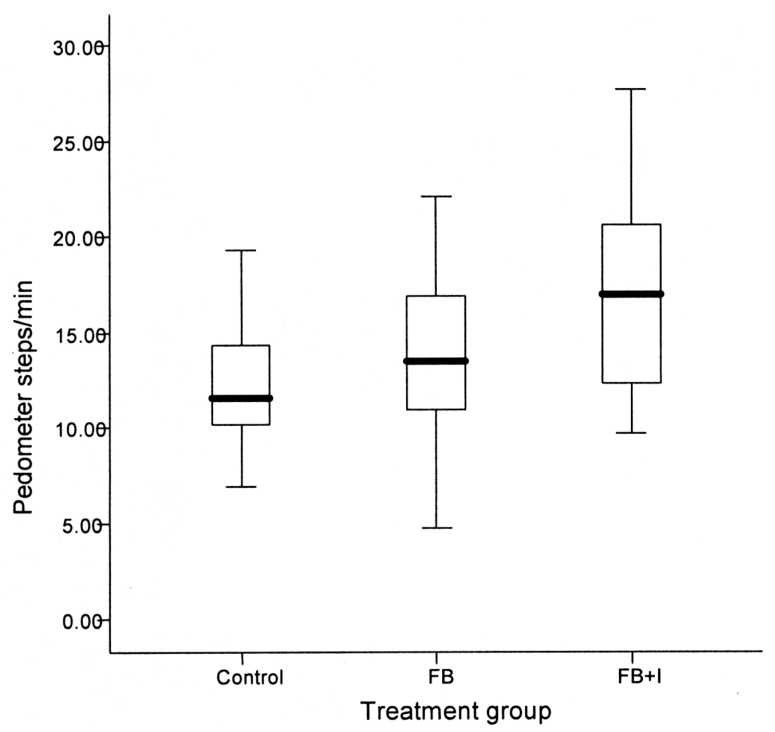

Figure 1 - Boxplot showing the differences in pedometer steps/min by treatment group. Note. $\mathrm{FB}=$ feedback; $\mathrm{FB}+\mathrm{I}=$ feedback plus information. 


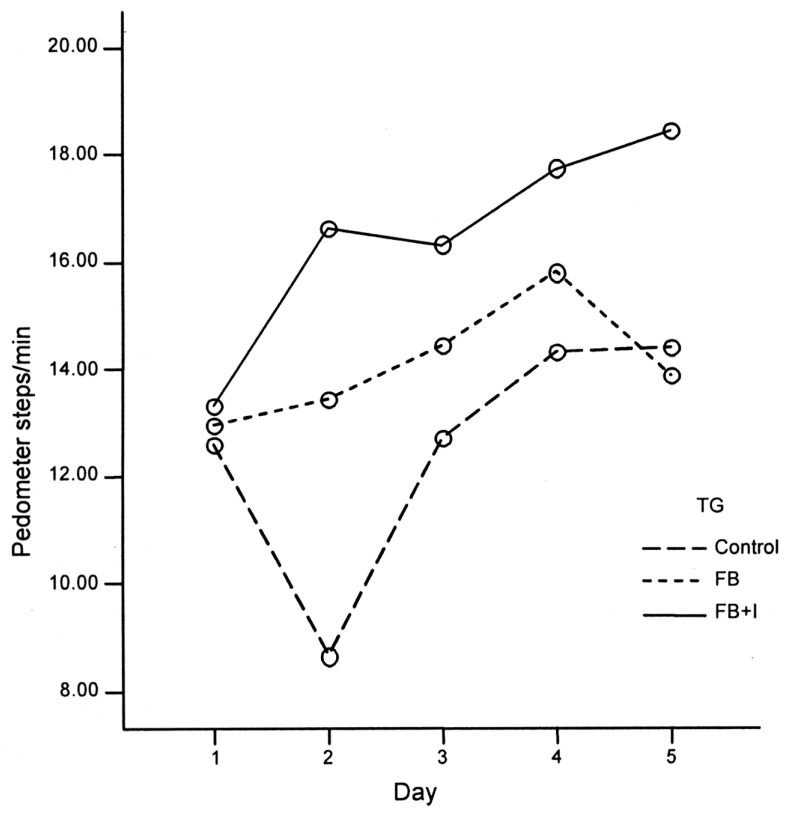

Figure 2 - Pedometer steps $\cdot \min ^{-1} \cdot$ day $^{-1}$ by treatment group. Note. FB = feedback; $\mathrm{FB}+\mathrm{I}=$ feedback plus information.

ferences in steps/min between Days 1 and $4(p=.012), 1$ and $5(p=.002), 2$ and $4(p=.014)$, and 2 and $5(p=.004)$.

\section{Discussion}

This study examined the effects of providing elementary-school students with feedback and information relating to the number of pedometer steps accumulated during a full school day. The FB+I group achieved significantly more steps than the FB and CON groups, whose step values were similar. This suggests that feedback alone is not sufficient to significantly increase students' pedometer step counts over the course of the school day. Information might therefore be required to facilitate students' PA competence and intrinsic motivation in order to improve their steps $(10,14,31)$. The information the students and teachers received on how to increase the number of steps students took focused on different opportunities during the school day, namely recess (24) and PE (9). These periods provide optimal opportunities for students to be active when not in classroom-based lessons. Because previous studies have found that PA decreases during wet weather as a result of students not being allowed outside at recess (21), the information also included suggestions on physical activities to do when children were required to remain indoors.

Each group demonstrated an increase in steps from Day 1 to Day 5. This was hypothesized to occur in the FB and FB+I groups, because students were asked to try 
to increase the number of steps taken each day. The increase was not expected from the CON group, however, which suggests that reactivity to wearing the pedometer might have occurred. This is contrary to results of two previous studies that have explored reactivity $(21,26)$. Both of those studies were conducted with students of ages similar to those in the present study but suggested that reactivity does not occur when pedometers are sealed. Despite the CON group being instructed to go about their normal activities at school, any reactivity might have been a result of the student's awareness of the importance of PA in relation to health and his or her knowledge of the use of pedometers. This basic appreciation of the utility of pedometers was observed during informal discussions with the students during the data-collection period. For example, many of them were able to explain the use of pedometers and recommended daily steps for health publicized in a recent media health-promotion campaign. A period of familiarization with wearing the pedometers before the intervention might have reduced the reactivity and should be considered in future research.

For each group there was 1 day when the number of steps noticeably decreased. For the CON (Day 2) and FB+I (Day 3) groups, these were identified as wet-weather days when children were prohibited from going outside during recess. In their study on reactivity in pedometers, Ozdoba et al. (21) also found that rainy days elicited a reduction in step counts. Paired $t$ tests, however, revealed that between the wet and previous days, the reduction in steps/min in the $\mathrm{FB}+\mathrm{I}$ group $(t=0.694, p=.490)$ was less marked than that of the CON group $(t=4.460, p=.0001)$. This suggests that the information and ideas given to the $\mathrm{FB}+\mathrm{I}$ group helped them achieve step counts greater than the baseline level, even though they were denied the opportunity to go outdoors at recess. Other studies that have adopted the "moving school" approach (4), which focuses on behavioral influences and class organization, such as group work and dynamic seating, have reported significant increases in PA during the school day, demonstrating that PA can be increased inside the school building.

Data were collected between the end of December and the beginning of April because of time constraints associated with the schools. Under more favorable circumstances data collection would have occurred simultaneously in each school. There was relatively little difference, however, in the average temperature and rainfall over this period (December temperature $=6.9^{\circ} \mathrm{C}$, rainfall $=64 \mathrm{~mm}$; April temperature $=9.6^{\circ} \mathrm{C}$, rainfall $\left.=74 \mathrm{~mm}\right)$. Furthermore, recent work conducted by Ridgers et al. (23) in schools from the same city and socioeconomic background as those in our study found that there were no significant differences in the day-today and season-to-season variability of children's PA during recess. On this basis they suggested that there might be no need to correct for seasonal differences in PA during recess, which was the period of time when we hypothesized that most PA would occur. Accordingly, we are confident that the students' step counts were not adversely affected by the timing of the data collection.

The reduction of step counts on Day 5 for the FB group cannot be explained by weather conditions. It is possible that these students reached a ceiling of how active they could be without any additional information, ideas, or stimuli beyond the pedometer step-count feedback. As a result, this group might have become disinterested in the pedometer target by the fifth day if they felt unable to further enhance their activity levels. The premise for this supposition is that promotion and maintenance of children's PA depend on the development of perceived competence 
and intrinsic motivation $(10,14,31)$. Had the intervention period extended beyond 5 school days, a lack of improvement on the fifth day evidenced by decreased step-count feedback could have negatively affected some FB students' PA competence and, therefore, their intrinsic motivation to continue to improve. This might be important for future interventions designed to increase children's PA by using feedback as a basis for enhancing competence perceptions.

Providing feedback and information appeared to be a successful approach, regardless of gender, suggesting that girls and boys were equally receptive to the feedback and information provided. Overall, however, boys were generally more active than girls. This concurs with previous findings by Ridgers et al. (24), who assessed children's PA during recess using accelerometry. It was reported that boys were almost $10 \%$ more active than girls during recess, possibly as a result of the social context of the playground and the types of activities in which both genders chose to engage. Boys tended to dominate the area and take part in more vigorous activities such as ball games (24), which would be likely to register pedometer steps. Conversely, girls generally participated on the periphery of the playground in lighter, less dynamic activities (24), which would most probably result in fewer pedometer steps. Given that recess provides the most frequent opportunity for children to be active at school, these sociocultural factors might be a plausible reason for the gender differences observed in the present study.

This study was limited by the sample size and the short duration of the intervention. Although an acute effect was demonstrated, an intervention sustained over a longer period would provide a better indication of treatment effect. Furthermore, longitudinal designs might examine the possible seasonal variability of students' PA more effectively.

\section{Conclusions}

This study demonstrated that pedometers can provide feedback to facilitate a shortterm increase in students' pedometer steps during the school day. Feedback from pedometers alone was not sufficient to elicit a statistically significant increase in steps, and it is likely that providing additional PA information can avoid intervention fatigue. Simple strategies such as the one used in this study can be applied to increase children's PA in school settings. Pedometers present a relatively affordable means of promoting PA. Purchasing large numbers of "research-standard" pedometers (e.g., New Lifestyles Digiwalker SW-200), however, might be beyond the fiscal means of most schools. Less expensive pedometers, such as the one used in this study, might be more affordable tools for schools wishing to independently measure PA as an educational method of promoting PA and health among their students.

\section{References}

1. Beets, M.W., M.M. Patton, and S. Edwards. The accuracy of pedometer steps and time during walking in children. Med. Sci. Sports Exerc. 37(3):513-520, 2005.

2. Beighl, A., R.P. Pangrazzi, and S.D. Vincent. Pedometers, physical activity and accountability. J. Phys. Educ. Recreation Dance. 72:16-25, 2001.

3. Biddle, S., K.R. Fox, and S.H. Boutcher. Physical Activity and Psychological WellBeing. New York, NY: Routledge, 2000. 
4. Cardon, G., D. De Clercq, I. Bourdeaudhuij, and D. Breithecker. Sitting habits in elementary schoolchildren: a traditional versus a ' $\square$ oving school.' Patient Educ. Couns. 54(2):133-42. 2004.

5. Cole, T.J., M.C. Bellizzi, K.M. Flegal, and W.H. Dietz. Establishing a standard definition for child overweight and obesity worldwide: international survey. BMJ. 320:1-6, 2000.

6. Corbin, C. B., and R.P. Pangrazi (Eds.). Physical activity for children: a statement of guidelines. J. Phys. Educ. Recreation Dance. 3-16, 1999.

7. Docheff, D.M. The feedback sandwich. J. Phys. Educ. Recreation Dance. 61:17-18, 1990.

8. Epstein, L.H., R.A. Paluch, L.E. Kalakanis, G.S. Goldfield, F.J. Cerny, and J.N. Roemmich. How much activity do youth get? A quantitative review of heart rate measured physical activity. Pediatrics. 108:1-10, 2001.

9. Fairclough, S.J., and G. Stratton. Physical education makes you fit and healthy: physical education's contribution to young people's physical activity levels. Health Educ. Res. 20:14-23, 2005.

10. Ferrer-Caja, E., and M.R. Weiss. Predictors of intrinsic motivation among adolescent students in physical education. Res. Q. Exerc. Sport. 71:267-279, 2000.

11. Fox, K.R., A. Cooper, and J. McKenna. The school promotion of children's healthenhancing physical activity: perspectives from the United Kingdom. J. Teach. Phys. Educ. 23:336-355, 2004.

12. Fuentes, R.M., I.L. Notkola, S. Shemeikka, J. Tuomilehto, and A. Nissinen. Tracking of body mass index during childhood: a 15-year prospective population based family study in eastern Finland. Int. J. Obes. 27:716-721, 2003.

13. Gavarry, O., M. Giacomoni, T. Bernard, M. Seymat, and G. Falgairette. Habitual physical activity in children and adolescents during school and free days. Med. Sci. Sports Exerc. $\square 525-531,2003$.

14. Harter, S. The determinants and mediational role of global self-worth in children. In: Contemporary Topics in Developmental Psychology. N. Eisenberg (Ed.). New York, NY: Wiley, 1987, pp. 219-242.

15. Kang, M., W. Zhu, C. Tudor-Locke, and B.E. Ainsworth. An experimental determination of the best missing-value recovery method in assessing physical activity using pedometers. Med. Sci. Sports Exerc. 27:1326-1332, 2003.

16. Keiss, W., A.G. Galler, A. Reich, et al. Clinical aspects of obesity in childhood and adolescents. Obes. Rev. 2:29-36, 2001.

17. Lohman, T.G., A.F. Roche, and R. Martorell. Anthropometric Standardization Reference Manual. Champaign, IL: Human Kinetics, 1988.

18. McKenzie, T.L., S.J. Marshall, J.F. Sallis, and T.L. Conway. Leisure-time physical activity in school environments: observational study using SOPLAY. Am. J. Prev. Med. 30:70-77, 2000.

19. Michaud-Tomson, L., and M. Davidson. Walk to school-does it make a difference in children's physical activity levels? ACHPER — 50:16-24, 2003.

20. Ozdoba, R., C. Corbin, and G. Le Masurier. Does reactivity exist in children when measuring physical activity with unsealed pedometers? Pediatr. Exerc. Sci. 16:158-166, 2004.

21. Riddoch, C.J., L. Bo Andersen, N. Wedderkopp, et al. Physical activity levels and patterns of 9- and 15-yr-old European children. Med. Sci. Sports Exerc. 36:86-92, 2004.

22. OFSTED. 2006. Available at: http://www.ofsted.gov.uk/. Accessed February 2006.

23. Ridgers, N.D., G. Stratton, E. Clarke, S.J. Fairclough, and D.J. Richardson. Day-tosay and seasonal variability of physical activity during school recess. Prev. Med. In press.

24. Ridgers, N.D., G. Stratton, and S.J. Fairclough. Assessing physical activity during recess using accelerometry. Prev. Med. 1:102-7, 2005. 
25. Rogers, E., A.M. Moon, M.A. Mullee, V.M. Speller, and P.J. Roderick. Developing the 'healthy-promoting school' - a national survey of healthy schools award. BMC Public Health. 112:37-40, 1998.

26. Rowe, D.A., M.Y. Mahar, T.D. Raedeke, and J. Lore. Measuring physical activity in children with pedometers: reliability, reactivity and replacement of missing data. Pediatr. Exerc. Sci. 16:343-354, 2004.

27. Shomaker, K.E. The effect of pedometers on motivation and steps in fitness walking classes. KinPubs. 43-45, 2003.

28. Tudor-Locke, C., and C. Corbin. Taking steps toward increased physical activity: using pedometers to measure and motivate. PCPFS. 3:1-8, 2002.

29. Tudor-Locke, C., J.E. Williams, J.P. Reis, and D. Pluto. Utility of pedometers for assessing physical activity. Sports Med. 32:795-808, 2002.

30. Vincent, S.D., and C.L. Sidman. Determining measurement error in digital pedometers. Meas. Phys. Ed. Exerc. Sci. 7:19-42, 2003.

31. Weiss, M.R., C. Corbin, and B. Pangrazzi. Motivating kids in physical activity. PCPFS. 口 $3: 1-7,2000$. 